\title{
Mortalidad por aborto séptico en el Hospital Nacional Cayetano Heredia 1985-1992
}

\author{
Mortality due to septic abortion at the Hospital Nacional Cayetano Heredia
}

\section{Castro Raúl, Maradiegue Eduardo ${ }^{1}$}

${ }^{1}$ Jefe de Departamento de Obstetricia y Ginecología, Hospital Nacional Cayetano Heredia.

\section{RESUMEN}

Estudio retrospectivo, transversal, clínico, tipo caso control de 24 muertes por aborto séptico, comparadas con 72 controles escogidas mediante un muestreo sistemático de la población que no falleció. La tasa de mortalidad por aborto séptico para el periodo 1985-1992 fue de 67.3 por 100,000 nv., la más alta se obtuvo para 1991 con 176.6. el 42\% de ingresos a Ginecología fu aborto, siendo el $8 \%$ séptico. Los factores de riesgo para mortalidad fueron 5 o más gestaciones $(\mathrm{OR}=1.7)$, edad gestacional mayor de 16 semanas $(\mathrm{OR}=5.0)$, tiempo de maniobras abortivas mayor de 5 días $(\mathrm{OR}=1.7)$, shock séptico $(\mathrm{OR}=8.5)$, anemia $(\mathrm{OR}=3.4)$, insuficiencia renal aguda $(\mathrm{OR}=17.0)$, perforación uterina $(\mathrm{OR}=5.5)$, coagulación intravascular diseminada $(\mathrm{OR}=60.0)$, tromboflebitis pélvica $(\mathrm{OR}=10.2)$, falla multiorgánica $(0 \mathrm{R}=6.5)$ y pulmón de shock $(\mathrm{OR}=6.5)$. Los síntomas y signos con diferencias significativas fueron secreción maloliente, ictericia, petequias, disnea y mialgia. Para tratamiento médico encontramos transfusión, plasma, cardiotónicos y anticoagulantes, siendo para el quirúrgico histerectomía abdominal total más salpingooforectomía bilateral. Las principales causas de muerte fueron shock séptico, insuficiencia renal aguda, falla multiorgánica, coagulación intravascular diseminada y tromboembolia pulmonar. (Rev Med Hered 1994; 5; 154-160)

PALABRAS CLAVE: Mortalidad, aborto, shock, séptico.

\section{SUMMARY}

A retrospective, transversal, clinic, epidemiological, type control case study of twenty-four septic abort deaths was done in the HNCH between 1985 and 1992. The control group was 
formed by seventy-two patients choosen from the pregnant population that didn't die. The septic abort mortality rate for the period in the study was 67.3 for 100,000 life birth, the most elevated annual rate was calculated in 1991 whit 176.6. The riskfactor for mortality is 5 or more pregnancies $(\mathrm{OR}=1.7)$, gestation age more 16 weeks actue renal failure $(\mathrm{OR}=17)$, uterine perforation $(\mathrm{OR}=5.5)$ disseminate intravascular coagulation $(\mathrm{OR}=60.0)$, pelvic trombophlebitis $(\mathrm{OR}=10.2)$, multiorganic failure $(\mathrm{OR}=6.5)$ and lung shock $(\mathrm{OR}=6.5)$.

The symptoms and signs with significant difference decay secretion, ykellowish, stain red, grief and pain muscle. For medical treatment was transfution, plasma, cardiotonics and anticoagulation, for surgical HAT + SOB. The causes of death were septic shock, acute reanal failure, multiorganic failure,disseminate intravascualr coagulation and lung tromboembolus.(Rev Med Hered 1994; 5:154-160).

KEY WORDS: Mortality, abort, shock, septic.

\section{INTRODUCCION}

Las implicancias socio económicas, culturales y éticas que involucran al aborto, así como las graves complicaciones y secuelas que provoca, hacen de éste un problema de salud pública sobre todo en países en desarrollo donde su incidencia es alta, independiente de lugar, tiempo o raza $(1,2,3,4,5,6,7,8)$.

El aborto séptico sigue siendo expresión de una desigual situación socio-económica y cultura la involucrar principalmente a mujeres de sectores marginados de la producción, desprotegidas de la seguridad social y con bajos ingresos lo que generalmente origina desnutrición, hacinamiento e ignorancia, factores importantes en la predisposición a la infección $(13,14,15,16,17,18)$.

Hall (19) en 1965 a nivel nacional reporta una tasa de incidencia de 175.0 por $1000 \mathrm{nv}$. y Sánchez (20) en 1967 en el IPSS 178.0 por 1000 nv.

El Ministerio de Salud para el período 1977-78 informa para sus 97 establecimientos una tasa de 137.0 por 1000 nv. que aumentó significativamente a 200.0 cuando Castello (10) en 1987 hace su reporte a nivel nacional. La ilegalidad del aborto hace difícil precisar la real magnitud de este, ya que sólo acuden a los hospitales aquellos que presentan complicaciones; infección o hemorragia. Sin embargo los datos obtenidos suministran una importante fuente de información al revelar el funcionamiento del sistema médico en su conjunto, permitiendo fijar los lineamientos de un eficaz programa preventivo, promocional para la disminución de su tasa $(10,21,22)$.

Rechkemmer (8) encuentre en el Hospital Arzobispo Loayza para el periodo 1978-1982 una tasa de mortalidad por aborto séptico de 406.0 por 100,00nv., luego Maradiegue (23) reporta en el Hospital Nacional Cayetano Heredia la tasa de 714.0 para el comprendido entre 1975-1984.

Este estudio tiene como objetivo actualizar la tasa de mortalidad por aborto séptico en la población que acudió al Hospital Nacional Cayetano Heredia entre el $1^{\circ}$ de enero de 1985 y el 31 de diciembre de 1992 y determinar los factores de riesgo. 


\section{MATERIAL Y METODOS}

Estudio retrospectivo, observacional, transversal, clínico, epidemiológico sobre mortalidad por aborto séptico en el Hospital Nacional Cayetano Heredia $(\mathrm{HNCH})$ de la población que acudió entre $1^{\circ}$ de enero al 31 de diciembre 1992.

Criterios de inclusión de casos y controles

Paciente fallecida con diagnóstico de aborto séptico, y paciente con aborto séptico dque no fallece

Estudio estadístico

A las variables discretas se las analizó mediante la prueba de Chi Cuadrado Y cuando el número de casos más controles era menor de 2.5 se aplicó la prueba de Fisher. Para las variables continuas se usó el análisis de varianza. El nivel de significancia utilizado fue del 95\%. Se calculó el Odds Ratio (OR) en los que se encontró diferencia estadísticamente significativa.

\section{RESULTADOS}

La tasa de mortalidad materna por aborto séptico en el HNCH para el periodo 1985-1992 fue de 67.3 por $100,000 \mathrm{nv}$. la mas alta se obtuvo para 1991 con 176.6. El $42.0 \%$ de ingresos a Ginecología fue por aborto. El 8.0\% del total de abortos son sépticos (cuadro No 1 ).

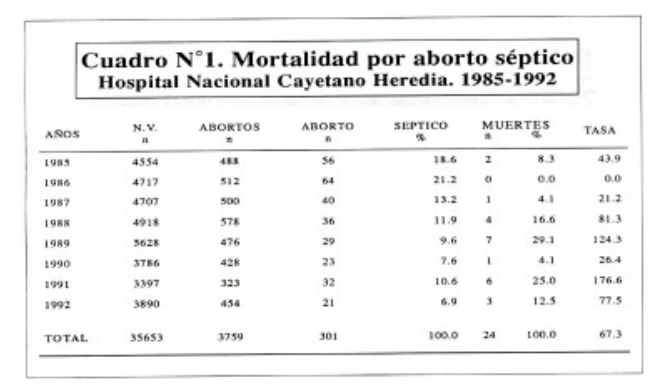

La edad del $58.3 \%$ de casos y $72.2 \%$ de controles estuvo comprendida entre 21 y 24 años. El $33.3 \%$ de casos fueron primigestas, correspondiendo $45.8 \%$ para pacientes con 5 o más gestaciones mientras que las cifras encontradas para sus controles fueron 19.4 y $31.9 \%$ respectivamente. La diferencia fue significativa para 5 o más gestaciones $(\mathrm{OR}=1.7)$. El control pre-natal deficiente se presentó en el 91,6\% de casos y 95,8\% de controles (cuadro № 2). 


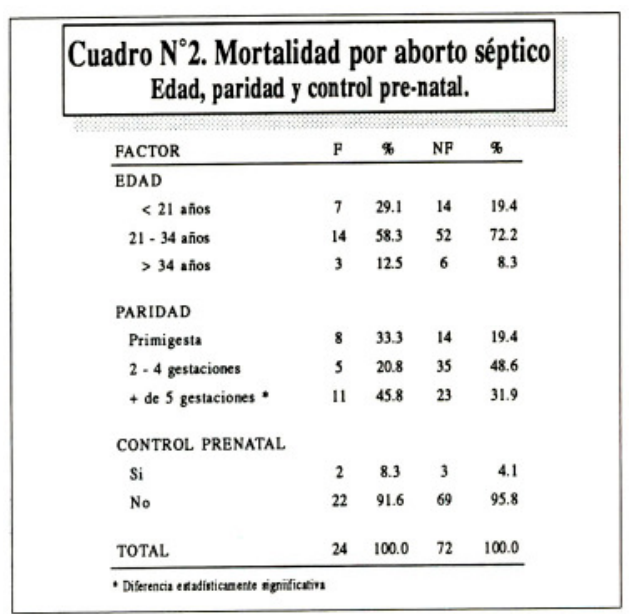

Al analizar la edad gestacional hallamos que el mayor porcentaje tanto para casos $(49.9 \%)$ y controles (55.4\%) estuvo comprendido entre la 5ta. Y 10ma. Semana. Se encontró diferencia significativa para pacientes con un tiempo de gestación mayor de 16 semanas $(\mathrm{OR}=5.0)$ (Cuadro No3).

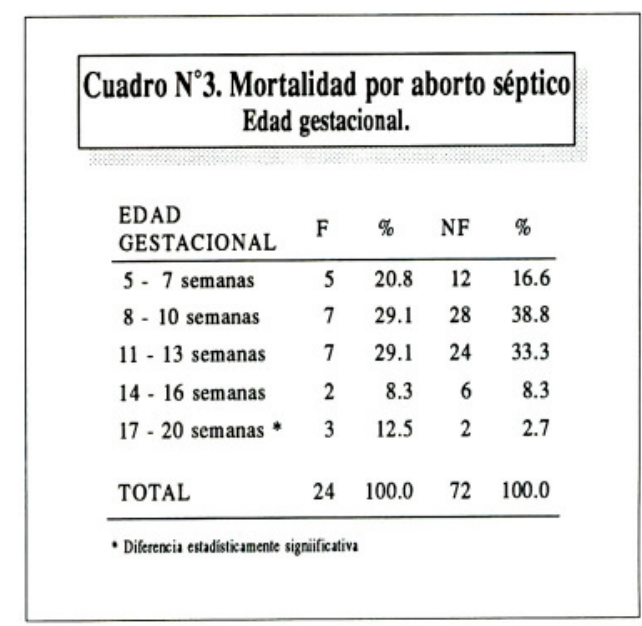

El cuadro $N^{o} 4$ muestra el tiempo de maniobras abortivas al ingreso, observamos que ningún caso acudió durante las primeras 24 horas post-aborto en contraste al $16.9 \%$ de controles que si lo hicieron. El 53.7 y $39.5 \%$ de casos y controles tuvieron un tiempo de maniobras mayor de 5 días $(\mathrm{OR}=1.7)$, siendo la diferencia significativa. 


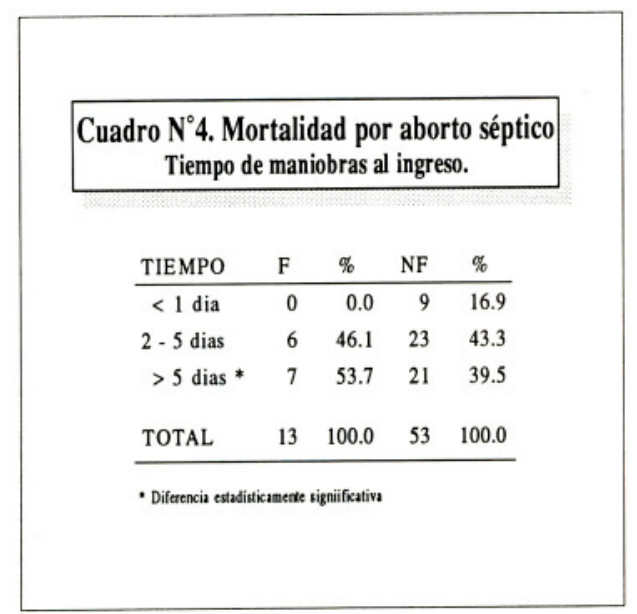

Los principales síntomas para los casos fueron fiebre $(62,5 \%)$, dolor hipogástrico $(62,5 \%)$ y escalofríos $(41,6 \%)$ correspondiendo a sus controles $73.6,79.1$ y $25 \%$ respectivamente. Se obtuvo diferencia significativa para disnea y mialgia. Los principales signos Ginecorragia $(75,0 \%)$ y secreción maloliente $(75,0 \%)$. que para sus controles correspondió 89.9 y 38,8\% (Cuadro No5). Fueron síntomas significativos: secreción maloliente, ictericia y petequias.

\begin{tabular}{|c|c|c|c|c|}
\hline \multicolumn{5}{|c|}{$\begin{array}{c}\text { Cuadro } \mathbf{N}^{\circ} \text { 5. Mortalidad por aborto séptico } \\
\text { Síntomas y signos al ingreso. }\end{array}$} \\
\hline & $\mathrm{F}$ & $\%$ & NF & $\%$ \\
\hline \multicolumn{5}{|l|}{ SINTOMAS } \\
\hline Dolor hipogástrico & 15 & 62.5 & 57 & 79.1 \\
\hline Fiebre & 15 & 62.5 & 53 & 73.6 \\
\hline Escalosfrios & 10 & 41.6 & 18 & 25.0 \\
\hline Nauseas y vómitos & 5 & 20.8 & 15 & 20.8 \\
\hline Malestar general & 5 & 20.8 & 13 & 18.0 \\
\hline Dísnea * & 4 & 16.6 & 1 & 1.3 \\
\hline Mialgia * & 3 & 12.5 & 0 & 0.0 \\
\hline Cefalea & 1 & 4.1 & 6 & 8.3 \\
\hline Dolor pélvico & 1 & 4.1 & 4 & 5.5 \\
\hline \multicolumn{5}{|l|}{ SIGNOS } \\
\hline Ginecorragia & 18 & 75.0 & 59 & 89.9 \\
\hline Secreción maloliente * & 18 & 75.0 & 28 & 38.8 \\
\hline Ictericia * & 5 & 20.8 & 1 & 1.3 \\
\hline Pérdida de líquido & 3 & 12.5 & 3 & 4.1 \\
\hline Petequias * & 3 & 12.5 & 0 & 0.0 \\
\hline Oliguria & 1 & 4.1 & 1 & 1.3 \\
\hline TOTAL & 24 & 100.0 & 72 & 100.0 \\
\hline
\end{tabular}


La principal complicación de pacientes que no manifestaron, para los casos fue shock séptico $(70.0 \%)$ y para los controles pelviperitonitis $(46,3 \%)$. Para los casos que manifestaron maniobras fue shock séptico(76,9\%) para controles fue anemia $(35.8 \%)$. La diferencia fue significativa para shock séptico $(\mathrm{OR}=8.59)$, anemia $(\mathrm{OR}=3.4)$, IRA $(\mathrm{OR}=17.0)$, perforación uterina $(\mathrm{OR}=5.5), \mathrm{CID}(60.0)$, tromboflebitis pélvica $(\mathrm{OR}=10.1)$, pulmón de shock $(\mathrm{OR}=6.5)$ y $\mathrm{FMO}(\mathrm{OR}=6.5)$. (Cuadro $\left.\mathrm{N}^{\mathrm{o}} 6\right)$.

\section{Cuadro $\mathrm{N}^{\circ}$ 6. Complicaciones según confesión de maniobras}

\begin{tabular}{|c|c|c|c|c|c|c|c|c|c|c|c|c|}
\hline \multirow[b]{2}{*}{ COMPLICACION } & \multicolumn{4}{|c|}{ NO CONFESARON } & \multicolumn{4}{|c|}{ CONFESARON } & \multicolumn{4}{|c|}{ TOTAL } \\
\hline & $\mathrm{F}$ & $\%$ & NF & $\%$ & $\mathrm{~F}$ & $\%$ & NF & $\%$ & F & $\%$ & NF & $\%$ \\
\hline Shock séptico * & 7 & 70 & 4 & 21.0 & 10 & 76.9 & 12 & 22.6 & 17 & 70.8 & 16 & 22.2 \\
\hline Anemia * & 5 & 50 & 2 & 10.5 & 9 & 69.2 & 19 & 35.8 & 14 & 58.3 & 21 & 29.1 \\
\hline Pelviperitonitis & 4 & 40 & 9 & 47.3 & 4 & 30.7 & 11 & 20.7 & 8 & 33.3 & 20 & 27.7 \\
\hline IRA * & 3 & 30 & 2 & 10.5 & 9 & 69.2 & 2 & 3.7 & 12 & 50.0 & 4 & 5.5 \\
\hline Perforación uterina * & 3 & 30 & 3 & 15.7 & 5 & 38.4 & 3 & 5.6 & 8 & 33.3 & 6 & 8.3 \\
\hline CID * & 2 & 20 & 1 & 5.2 & 9 & 69.2 & 0 & 0.0 & 11 & 45.8 & 1 & 1.3 \\
\hline Lacer. cervix & 2 & 20 & 3 & 15.7 & 0 & 0.0 & 4 & 7.5 & 2 & 8.3 & 7 & 9.7 \\
\hline Tromb. pélvica * & 2 & 20 & 0 & 0.0 & 1 & 7.6 & 0 & 0.0 & 3 & 12.5 & 0 & 0.0 \\
\hline FMO * & 1 & 10 & 0 & 0.0 & 1 & 7.6 & 0 & 0.0 & 2 & 8.3 & 0 & 0.0 \\
\hline Hemoperitoneo & 0 & 0 & 1 & 5.2 & 0 & 0.0 & 1 & 1.8 & 0 & 0.0 & 2 & 2.7 \\
\hline Shock hipovolémico & 0 & 0 & 1 & 5.2 & 1 & 7.6 & 2 & 3.7 & 1 & 4.1 & 3 & 4.1 \\
\hline Panmetritis & 0 & 0 & 2 & 10.5 & 2 & 15.3 & 0 & 0.0 & 2 & 8.3 & 2 & 2.7 \\
\hline Pulmón de shock * & 1 & 10 & 0 & 0.0 & 1 & 7.6 & 0 & 0.0 & 2 & 8.3 & 0 & 0.0 \\
\hline Ab. fetal tardío & 1 & 10 & 1 & 5.2 & 0 & 0.0 & 0 & 0.0 & 1 & 4.1 & 1 & 1.3 \\
\hline TOTAL & 11 & 100 & 19 & 100.0 & 13 & 100.0 & 53 & 100.0 & 24 & 100.0 & 72 & 100.0 \\
\hline
\end{tabular}

Los principales tratamientos médicos empleados para los casos fueron hidratación $(100,0 \%)$ y antibióticos (95,8\%), las cifras halladas para sus controles fueron 100.0 y $100,0 \%$ respectivamente.

Se encontró diferencia significativa para transfusión, plasma, plaquetas, asi como para cardiotónicos.

Para tratamiento quirúrgico la diferencia fue significativa para $\mathrm{HAT}+\mathrm{SOB}$.(Cuadro $\mathrm{N}^{\mathrm{o}}$ 7). 


\begin{tabular}{|c|c|c|c|c|}
\hline \multicolumn{5}{|c|}{$\begin{array}{c}\text { Cuadro } \mathbf{N}^{\circ} \text { 7. Mortalidad por aborto séptico } \\
\text { Tratamiento. }\end{array}$} \\
\hline . & $\mathrm{F}$ & \% & NF & 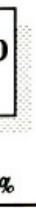 \\
\hline \multicolumn{5}{|l|}{ TRATAMIENTO MEDICO } \\
\hline Hidratación & 24 & 100.0 & 72 & 100.0 \\
\hline Transfusión * & 9 & 37.5 & 0 & 0.0 \\
\hline Plasma * & 4 & 16.6 & 0 & 0.0 \\
\hline Plaquetas * & 1 & 4.1 & 0 & 0.0 \\
\hline Corticoides & 3 & 12.5 & 0 & 0.0 \\
\hline Cardiotónicos * & 18 & 75.0 & 0 & 0.0 \\
\hline Anticoagulantes * & 2 & 8.3 & 0 & 0.0 \\
\hline Hematínicos & 10 & 41.6 & 14 & 19.4 \\
\hline Oxitócicos & 15 & 62.5 & 46 & 63.8 \\
\hline Analgésicos & 20 & 83.3 & 61 & 84.7 \\
\hline Diuréticos & 3 & 12.5 & 0 & 0.0 \\
\hline Antibióticos & 23 & 95.8 & 72 & 100.0 \\
\hline $\mathrm{PEN}+\mathrm{CAF}+\mathrm{GEN}$ & 19 & 79.1 & 59 & 81.9 \\
\hline TRATAMIENTO MEDICO + CIRUGIA & 24 & 100.0 & 72 & 100.0 \\
\hline $\mathrm{HAT}+\mathrm{SOB} *$ & 16 & 66.6 & 11 & 15.2 \\
\hline $\mathrm{HAT}+\mathrm{SOU} *$ & 3 & 12.5 & 11 & 15.2 \\
\hline HAT & 1 & 4.1 & 2 & 2.7 \\
\hline LU & 8 & 33.3 & 56 & 77.7 \\
\hline $\mathrm{LAP}+\mathrm{DREN}$ & 44 & 16.6 & 42 & 5.5 \\
\hline CULDOSCENT. & 12 & 50.0 & 61 & 84.7 \\
\hline APENDICEC. & 2 & 8.3 & 1 & 1.3 \\
\hline * Diferencia estadísticamente signiificativa & & & & \\
\hline
\end{tabular}

Las principales causas de muerte de los casos fueron shock séptico $(58,3 \%)$, IRA $(20,8 \%)$, FMO $(8,3 \%)$, CID $(8,3 \%)$ y TEP $(4,1 \%)$ (Cuadro N 8 ).

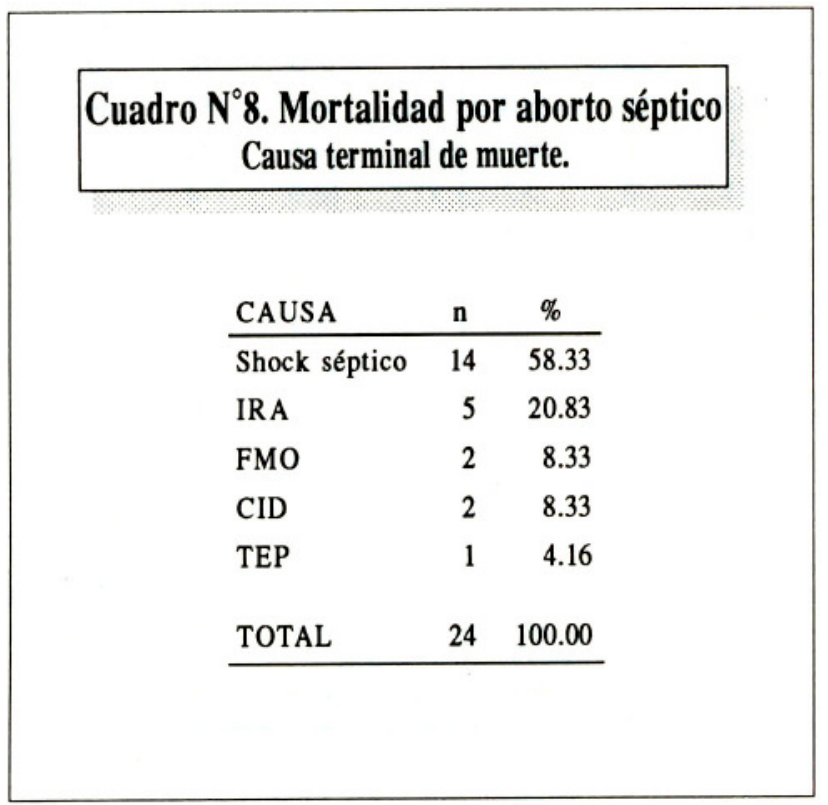




\section{DISCUSION}

A nivel mundial el aborto infectado representa una de las entidades infecciosas más frecuentes dentro de la Ginecología y Obstetricia. Por ser causa de graves complicaciones como shock séptico, insuficiencia renal aguda (IRA) coagulación intravascular diseminada (CID) y falla multiorgánica (FMO) entre otras, y su alta incidencia, se convierte en grave problema de salud pública, sobre todo en países en desarrollo.

En el Perú la tasa de mortalidad materna es más elevada que el promedio a nivel mundial y aún mayor que el latinoamericano.

La tasa de mortalidad por aborto séptico correlaciona con un número de variables socioeconómicas, las que interaccionan con factores médicos incrementando la predisposición a desarrollar complicaciones potenciales fetales.

En el HNCH Saona (24) en un estudio previo encontró que el $44.5 \%$ de ingresos al Servicio de Ginecología fue por aborto, cifra similar al $42 \%$ hallado en nuestra casuística.

Hay que considerar que un gran porcentaje de abortos no complicados son derivados por falta de camas de hospitalización, esta cifra se podría aumentar mediante un programa de tratamiento ambulatorio y alta precoz del aborto no infectado, tal como se reporta en la literatura $(25,26)$.

Es llamativo observar un alarmante incremento de pacientes que ingresan por aborto infectado y séptico; así como Gonzáles Enders (27) entre 1969 y 1972 reporta 10\% de abortos infectados y $1.5 \%$ de sépticos, posteriormente Saona (21) informa 25 y 4\% respectivamente para 1975 1984. Este reporte encuentra que el $27 \%$ del total de abortos son infectados, siendo el $8 \%$ sépticos. La tasa de mortalidad materna por aborto séptico para 1985-1992 fue de 67.3 por $100,000 \mathrm{nv}$.

Hay que considerar un notable aumento de la proporción de mujeres solteras que abortan, donde el 85\% manifestó maniobras abortivas (24).

El embarazo en estas mujeres no es aceptado por la sociedad por razones culturales, así este grupo recurre con mas frecuencia a empíricas incrementando el alto índice de abortos sépticos hallados $(27,28)$. Es resaltante señalar que en los últimos años la incidencia de aborto séptico ha aumentado en pacientes menores de 20 años, muestra de esto es la cifra de $29,1 \%$ encontrada para nuestros casos en contraste al $16.6 \%$ que reporta Saona (24) y que es 4 veces mayor al porcentaje encontrado por Villa Retamozo (35) en el Hospital Arzobispo Loayza, para 1970. Así un adecuado programa de educación secual impartido a jóvenes y adolescentes a diferentes niveles, debe formar parte de cualquier lineamiento general destinado a disminuir la tasa de mortalidad por aborto.

Para nuestros casos la proporción de abortos previos en mujeres mayores de 35 años fue del $100 \%$ lo que demuestra la baja información y uso de métodos anticonceptivos. Se halló que el $45,8 \%$ de caso y el $31,9 \%$ de controles tenían 5 o más gestaciones, siendo la diferencia significativa y el riesgo de muerte mayor.

Diversos reportes nacionales y extranjeros mencionan que entre el 58 y $66 \%$ de abortos se producen entre la 5ta. y 10ma. semana de gestación. Cervantes (29) reporta que el $24 \%$ de pacientes fallecidas por aborto tienen una edad gestacional entre 17 y 20 semanas y asocia 
incremento de mortalidad a mayor edad situación que ha sido demostrada por este y otros trabajos $(29,30,31)$.

Al haber realizado un procedimiento no aceptado por la sociedad y por el desconocimiento de la gravedad de su enfermedad las pacientes acuden tardíamente al hospital, así ningún caso acudió antes de 48 horas, en contraste al 16,9\% de sus controles que si lo hicieron. Gómez (34) encuentra una situación similar donde el mayor porcentaje de pacientes tenían un tiempo de maniobras abortivas entre 2 y 5 días, en este reporte encontramos diferencia significativa para un tiempo mayor de 5 días $(\mathrm{OR}=1.7)$.

En nuestras pacientes hallamos que el $75 \%$ de casos y el $38.8 \%$ de controles presentaron al ingreso secreción maloliente, siendo la diferencia significativa. Datos similares a los casos son reportados por Gutiérrez(32). Yamada (33) y Gómez (34).

Otros síntomas y signos con diferencia significativa fueron ictericia, petequias, disnea y mialgias.

Es llamativo encontrar algunas frecuencias más bajas que las informadas en otros estudios, así la fiebre que es citada entre 75 y $100 \%$ nosotros sólo la observación en $62.5 \%$, probablemente debido a la gravedad de las pacientes que se encontraban en la fase fría del shock séptico $(32,34)$.

La mortalidad fue mayor para las pacientes que cursaron con shock séptico $(\mathrm{OR}=8.5)$, anemia $(\mathrm{OR}=3.4)$, insuficiencia renal aguda $(\mathrm{OR}=17.0)$, perforación uterina $(\mathrm{OR}=5.3)$, coagulación intravascular diseminada $(\mathrm{OR}=60.0)$, falla multiorgánica $(\mathrm{OR}=6.5)$, pulmón de shock $(\mathrm{OR}=6.5)$ $\mathrm{y}$ tromboflebitis pélvica $(\mathrm{OR}=10.2)$.

El manejo actual aceptado por la mayoría de trabajos publicados incide en establecer incide en establecer en el diagnóstico en forma precoz, iniciar terapia antibiótica así como medidas de soporte y cirugía precoz $(12,27,32)$.

Para tratamiento médico encontramos diferencias significativa en el uso de transfusión, plasma, cardiotónicos y anticoagulantes.

La literatura en procesos infecciosos pélvicos severos, con compromiso multisistémico preconiza la remoción quirúrgica del foco séptico encontrándose supervivencia de hasta el $100 \%$ con posterior terapia hormonal de reemplazo. Todas nuestras pacientes se encontraban en la etapa reproductiva de tal forma que la ablación ovárica ocasiona menopausia precoz que amerita tratamiento. El procedimiento quirúrgico con significancia fue HAT=SOB (2).

Las tasas de mortalidad en pacientes que cursan con shock séptico son altas y en GinecoObstetricia tienen como principal causa al aborto séptico.

Gonzáles Enders(12). En 1973 en el HNCH lo reporta como causa terminal de 100\% de muertes, para nuestros casos alcanzó 58,3\%. En países desarrollados la infección como causa de muerte post-aborto ha disminuído y actualmente es reportada entre 20 y $25 \%$ incrementándose otras causas como problemas anestésicos y embolismo, lo que demuestra que ésta es evitable y es a su prevención a donde debemos dirigir todo nuestros esfuerzos (36). 


\section{Correspondencia:}

Dr. Eduardo Maradiegue

Departamento de Ginecología y Obstetricia, Hospital Nacional Cayetano Heredia.

Av. Honorio Delgado s/n San Martín de Porras

Lima-Perú.

\section{REFERENCIAS BIBLIOGRÁFICAS}

1.Barbato W. Aborto inducido: Epidemiología y profilaxis. Cuadernos Médicos Sociales . Asociación Médica de Rosario. CESS. Rosario Argentina. 1998.

2.Gutiérrez B. La ooforectomía en pelviperitonitis post-grado y post-parto. Tesis de especialista en G.O. Lima, Perú, UPCH. 194866 pp.

3.Konje JC. Health and economic consequences of septic inducid abortion. Int J Gynaecol. Obstet 1992; 37: 193-197.

4.Mascaro P, Bachman C. Mortalidad Materna en el Hospital Maternidad de Lima. Ginecol Obstet Peru 1985; 29:33-37.

5.Medina J. Aborto hospitalario en Bogotá. Rev Colomb Obstet Ginec 1985, 31: 40-3.

6.Purizaca M. Mortalidad Materna en el Hospital Regional Cayetano Heredia de Piura (19741981). Ginecol Obstet Peru 1982; 17: 19.

7.Rechet G. Inducet abortion and health problems in the developing countries. Lancet 1980; 11: 484-6.

8.Rechkemmer R. Aborto séptico. Tesis de Bachiller en Medicina, Lima-Perú UPCH 1983 $78 \mathrm{pp}$.

9.Binkin N. Tendencia de la mortalidad y morbilidad del aborto legal provocado. Ginecología y Obstetricia 1986; 1: 85-96.

10.Castello CJ. Diagnóstico de la situación de Salud en el Perú a nivel nacional. Trabajo del Gabinete de diagnóstico. UNMS Lima, 1987.

11.Díaz V, Muñoz C. Estudio del riesgo reproductivo en el Perú. Aspectos sociales: El aborto. Servicio sobre la enseñanza de la reproducción humana. Ica-Perú 12-15 de enero 1980.

12.Gonzáles Enders R. El aborto en el Perú: Problema de salud. Seminario Taller. Salud Materna y riesgo reproductivo. Aborto y esterilización. Sociedad Peruana de Ginecología y Obstetricia. Lima 1983.

13.Henhaw S. Aborto inducido una perspectiva mundial. Perspectivas Internacionales en Planificación Familiar. 1987. 
14.Sher N. Aborto como cuestión de Salud Pública. LILACS IV 1984, 163: 28-32.

15.OMS. Aborto espontáneo y provocado. Informe de un grupo cientif. Serie de informes técnicos \# 461. OMS Ginebra 1970.

16.OMS. Aborto provocado. Serie de informes técnicos. No 623. Ginebra 1978.

17.Pan American Health Organization. Investigación Interamericana de Mortalidad. Publicación Científica Setiembre 1967.

18.Pan American Health Organization. Epidemiology of abortion and practices of fertility regulation in latin America.Scientific Publication 306, Washington 1975.

19.Hall F. Planificación familiar. The milbank Memorial Fund Quarterly Vol. XLIII. New York 1965.

20.Sánchez K. La fertilidad de las mujeres limeñas . CEPD-Lima, 1967.

21.Castillo M. Salud Materno-Infantil. Morbilidad. Salud materna y riesgo reproductivo. Aborto y esterilización. Seminario Taller de la Sociedad Peruana de Ginecología y Obstetricia. Lima Perú. 14-15 de enero de 1980.

22.Ministerio de Salud. El aborto en los establecimientos de Salud del Perú. Dirección General de Salud Materno-Infantil. Ministerio de Salud. Lima, Perú 1980.

23.Maradiegue E. Mortalidd por aborto séptico en el HNCH(1975-1984). Acta Médica Peruana 1987, 14: 54-58.

24.Saona L. Epidemiología del aborto en el HNCH (1975-1984). Tesis de especialista en G.O. Lima, Perú, UPCH 1988.

25.Wangala S, Murgu M. Mortality due to abortion at Kenyatta National Hospital 1974-1983. Ciba Found Symp 198; 115: 4153.

26.Richard A. Lachman E. The incidence of major abdominal surgery after septic abortion and indicator of complications due to ilegal abortion . S Afr Med 1985; 68: 799-800.

27.Gonzáles Enders R. Shock séptico en Obstetricia y Ginecología. Tesis de Doctor en Medicina, Perú UPCH . 1973.

28.Alvo M, Kuzman C. Insuficiencia Renal Aguda: Experiencia de 22 años. Rev Med Chil 1981; 109: 420-427.

29.Cervantes R. Muerte materna intrahospitalaria: Análisis de 269 de los hospitales del Ministerio de Salud. Ginecol Obstet Perú 1987; 31: 41-48.

30.Hinojosa W. Aborto séptico en el Hospital Arzobispo Loayza. Complicaciones Médicas. Diagnóstico 1983; 12: 194-196. 
31.Pacheco J. Geisinger E. Mortalidad Materna en el IPSS. Ginecol Obst Perú, 1983; 28: 15.

32.Gutiérrez C. Infecciones severas post aborto. Diagnóstic 1987; 12: 194-197.

33.Yamada M. Aborto séptico e insuficiencia renal en el HNCH. Tesis de Bachiller, LimaPerú, UPCH. 1982; 58pp

34.Gómez D. Shock séptico en la enferma ginecológica del HNCH (1981-1991). Tesis de Bachiller en Medicina, Lima-Perú UPCH. 68pp.

35.Villa Retamozo O. Encuesta de aborto provocado. Tesis de Bachiller en Medicina, LimaPerú. UPCH. 1970; 53pp.

36.Pandit RD. Role of antenatal care in reducing maternal mortality. Asia Ocenanía J Obstet Ginaecol. 1992; 18: 1-6. 